\title{
Assessment of Thickened Renal Fascia as a Finding at Computed Tomography in the Diagnosis of Acute Pancreatitis
}

\author{
Parvin $\mathrm{R}^{1}$, Ali $\mathrm{MN}^{2}$, Sadeque ASQM ${ }^{3}$, Mohiuddin $\mathrm{AS}^{4}, \mathrm{Nahar}^{5}$
}

\begin{abstract}
This cross sectional study was carried out in the department of Radiology \& Imaging, BIRDEM, Dhaka, from January 2012 to December 2012 to find out the sensitivity of thickened renal fascia in diagnosis of acute pancreatitis at CT. Total 50 patients (mean age was 41.64 years with range from 18 to 65 years, 34M/ 16F) with clinical suspicion of acute pancreatitis included in this study after analyzing selection criteria. Total $32(64 \%)$ of the 50 clinically suspected cases were diagnosed as acute pancreatitis according to the criteria mentioned in material and methods. The mean thickness of renal fascia in acute pancreatitis was $7.20 \pm 3.32 \mathrm{~mm}$ (mean $\pm S D$ ) with range from 1 to $13 \mathrm{~mm}$ and maximum 18(40\%) patients were found having thickened fascia within 4.0 to $6.9 \mathrm{~mm}$. The extension of acute panceatitis only in left side was $71.8 \%$. In $21.8 \%$, the extension was bilateral. In $3.1 \%$ the renal fascial thickening was present on right side only. In the remaining 3.1\%, renal fascia was not thickened.In 31 (96.8\%) patients, the process extended into the anterior pararenal space. Both anterior and posterior renal fascia thickening was observed in 2(6.2\%) patients. Normal thickness of renal fascia was found in 1(3.1\%) patient. Sensitivity of thickened renal fascia in diagnosis of acute pancreatitis at CT was found 97\%, specificity $50 \%$, accuracy $80 \%$, positive predictive value $77 \%$, negative predictive value 90\%.It can be concluded that renal fascia thickening is an important as well as sensitive (97\%) CT feature in the diagnosis of acute pancreatitis.
\end{abstract}

1. Corresponding Author: Dr. Rehena Parvin Medical Officer

Department of Radiology and Imaging

Rangpur Medical College Hospital

2. Dr. Md. Naushad Ali

Assistant Professor

Department of Gastroenterology

Rangpur Medical College

3. Professor A.S.Q. Md. Sadeque

Director, Imaging Services

BIRDEM Hospital, Dhaka

4. Professor Dr. A.S. Mohiuddin

Head of the Department of Radiology and Imaging BIRDEM, Dhaka.

5. Dr. Nazmun Nahar

Assistant Professor

Department of Radiology and Imaging

Rangpur Medical College, Rangpur

\section{Introduction}

Acute pancreatitis is characterized by leakage and extravasation of activated pancreatic secretion. Acute pancreatitis leads to a wide range of local and systemic pathophysiologic alterations and to a large variability in the clinical manifestation and prognosis ${ }^{1}$. Acute pancreatitis accounts for $3 \%$ of all cases of abdominal pain admitted to the hospital. It affects 2-28/100 000 of population with an increasing incidence ${ }^{2}$. The mortality rate is only $1 \%$ in case of acute pancreatitis but it can approach $10-30 \%$ in case of severe pancreatitis. ${ }^{3}$ Overall mortality rate is $10-15 \%$ with $80 \%$ being self limiting ${ }^{4}$ ). Acute pancreatitis is reported to affect 5.4 to 79.8 per 100,000 population per year and $15 \%$ to $25 \%$ of them demonstrate a severe disease course. ${ }^{5}$ Most patients with acute pancreatitis who develop multiorgan failure have necrotizing pancreatitis and more than $80 \%$ of deaths occur in patients with pancreatic necrosis ${ }^{6}$. Acute pancreatitis is best defined clinically by a patient presenting with two of the following criteria:

1. Symptomps, such as epigastric pain consistent with the disease

2. A serum amylase or lipase greater than three times the upper limit of normal (reference range of amylase, 30-110 $\mathrm{U} / \mathrm{L}$ and serum lipase, 30-210 U/L); or

3. Radiological imaging consistent with the diagnosis, usually computed tomography or magnetic resonance imaging ${ }^{7}$.

For diagnosis of acute pancreatitis USG, CT, MRI, MRCP, ERCP, Plain $\mathrm{X}$ ray of abdomen, Barium Study, Percutaneous Pancreatography are used. USG is indicated early in an acute episode of pancreatitis, to help evaluate for the presence of gallbladder and/or common duct stones. Visualization of the pancreas is often impaired because of overlying bowel gas $^{8}$. MRI potentially offers some advantages over $\mathrm{CT}$ in acute pancreatitis, over and above radiation considerations. MRI has significant practical limitations in acutely ill patients ${ }^{9}$. Only CT scan among other procedures is used enormously in case of acute pancreatitis that not only can detect pancreatitis but also can score the severity of acute pancreatitis.

Several reports in the literature have described CT changes in pancreatic inflammatory disease, but little has been said about thickening of renal fascia secondary to pancreatitis.In acute pancreatitis, diffuse peripancreatic inflammation usually provoked. The extension of the peripancreatic inflammation is blocked by the Gerota's fascia. When peripancreatic inflammation spreads more 
extensively and becomes more severe do the inflammatory changes extend beyond the Gerota's fascia to the perirenal spaces $^{10}$

Imamura et at. (2010) introduce a new CT grading classification (Renal Rim Grade) of acute pancreatitis.RRG 1, no increase in the attenuation of the anterior pararenal and the perirenal spaces; RRG 2, increase in the attenuation of the pararenal space (pancreatic side of the Gerota's fascia) that is renal rim $\operatorname{sign}(+) ; R R G 3$, increase in the attenuation of both the pararenal and the perirenal spaces (renal side of the Gerota's fascia) that is loss of renal rim sign (+). When the perirenal bridging septa is thickened, RRG would be assessed as grade 3 .

In acute edematous pancreatitis, pancreas is apparently normal at CT but renal fascia is thickened. This thickened renal fascia is helpful in diagnosis. Renal fascia thickness is also useful and accurate clinically in severity assessment of acute pancreatitis. An early prediction of the prognosis of acute pancreatitis is possible by assessing the renal rim sign (the appearance of renal rim is called as renal rim sign $)^{5}$ Thus, by studying the thickened renal fascia even at noncontrast CT, diagnosis as well as prediction of prognosis of acute pancreatitis are possible. Thus we can guide the clinician to take early appropriate therapeutic decision which increase survival and enhanced quality of life. CT scan is painless procedure, which has well been accepted by both the patients and clinician. Thus, the purpose of this study is to find out the sensitivity of thickened renal fascia in diagnosis of acute pancreatitis at CT.

\section{Materials \& Methods}

The cross sectional study was carried out in the department of Radiology \& Imaging, BIRDEM for a period from January 2012 to December 2012. Patients with computed topographic findings of chronic pancreatitis, complications from prior episodes of pancreatitis, concomitant tumor and motion artifacts on the CT scan that limited evaluation were not included in this study. Patients less than thirteen years of age, trauma and post-operative patients were excluded. Thus, 50 patients having the clinical diagnosis of acute pancreatitis who came within the first 72 hours of onset of clinical symptoms were included in this study.

All the selected subjects underwent unenhanced and /or contrast-enhanced helical CT of abdomen. Demographic information was prospectively recorded and substantiated by means of inspection of medical records. Information included the subject's age, sex, and serum lipase and amylase level.

CT measurement of renal fascia thickness was done by researcher at first and findings were confirmed by consultant radiologist of the Department of Radiology and Imaging, BIRDEM who do not know patient's serum enzyme level to eliminate bias. Renal fascia thickness of one or both kidneys was recorted.Clinical history, laboratory findings of serum lipase and /or serum amylase, computed tomographic findings were collected in a pre-designed data collection sheet

\section{Result}

Total $32(64 \%)$ of the 50 clinically suspected cases were diagnosed as acute pancreatitis according to the criteria mentioned in material and methods.In this current study it was observed that the mean age was 41.64 years with range from 18 to 65 years and most of the patients (50\%) with acute pancreatitis were found in 4th decade. In this present study it was found that $68 \%$ and $32 \%$ were male and female respectively and male female ratio was almost 2.1:1.

The mean thickness of renal fascia in acute pancreatitis was $7.20 \pm 3.32 \mathrm{~mm}$ (mean $\pm \mathrm{SD}$ ) with range from 1 to $13 \mathrm{~mm}$ and maximum $18(40 \%)$ patients were found having thickned fascia within 4.0 to $6.9 \mathrm{~mm}$.

The extension of acute panceatitis only in left side was $71.8 \%$. In $21.8 \%$, the extension was bilateral. In $3.1 \%$ the renal fascial thickening was present on right side only .In the remaining $3.1 \%$, renal fascia was not thickened.In 31 (96.8\%) patients, the process extended into the anterior pararenal space. Both anterior and posterior renal fascia thickening was observed in $2(6.2 \%)$ patients. Normal thickness of renal fascia was found in $1(3.1 \%)$ patient.

Sensitivity of thickened renal fascia in diagnosis of acute pancreatitis was found $97 \%$, specificity $50 \%$, accuracy $80 \%$, positive predictive value $77 \%$, negative predictive value $90 \%$.

Table-1: Distribution of the acute pancreatitis patients according renal fascia thickness $(n=32)$.

\begin{tabular}{ccc}
\hline Renal fascia & Number of patients & Percentage \\
\hline Thick & 31 & $96.8 \%$ \\
Not thick & 1 & $3.1 \%$ \\
Total & 32 & $100 \%$ \\
\hline
\end{tabular}

Table 2: Sensitivity of thickened renal fascia at CT in the diagnosis of acute pancreatitis $(n=50)$

\begin{tabular}{cccc}
\hline $\begin{array}{c}\text { Thickened } \\
\text { renal fascia }\end{array}$ & $\begin{array}{c}\text { Acute } \\
\text { pancreatitic Patient }\end{array}$ & $\begin{array}{c}\text { Non-Acute } \\
\text { pancreatitic Patient }\end{array}$ & Total \\
\hline Present & $31(\mathrm{TP})$ & $9(\mathrm{FP})$ & 40 \\
Absent & $1(\mathrm{FN})$ & $9(\mathrm{TN})$ & 10 \\
Total & 32 & 18 & 50 \\
\hline
\end{tabular}

TP-True positive, TN-True negative, FP-False positive, FN-False negative, Sensitivity of thickened renal fascia is $97 \%$

\section{Discussion}

In this current study it was observed that the mean age was 41.64 years and most (50\%) of the patients with acute pancreatitis was found in 4th decade. Rithin et al. (2011) found the mean age was 40.9 years and the peak incidence of the disease was in the 4 th decade which is comparable 
with the current study. Bradley et al. (1989) showed average age of 41.3 years. In this present study it was observed that $68 \%$ and $32 \%$ were male and female respectively and male female ratio was almost 2.1:1 which is almost similar to the study of Imamura et al. (2010). Rithin et al. (2011), Chishty et al. (2005), Lenhart et al. (2008), Casas et al. (2004), Balthazar et al. (1990), Bradley et al. Murphy \& Ferguson (1989) showed male predominance in their study.

Total $32(64 \%)$ of the 50 clinically suspected cases were diagnosed as acute pancreatitis according to the criteria of Tenner \& Steinberg (2010) ${ }^{7}$.

In this current series it was observed that $31(96.8 \%)$ of these 32 acute pancreatitis patients had thickened renal fascia at CT. In the remaining $1(3.1 \%)$ case renal fascia was normal in thickness. Total $18(36 \%)$ of the 50 clinically suspected cases were not diagnosed as acute pancreatitis. ${ }^{9}(18 \%)$ of these 18 non pancreatitis patients had thickened renal fascia may be due to ischemic colitis or psoas abscess and renal fascia was found normal in thickness in remaining ${ }^{9}(18 \%)$ patients.

In $1(3.1 \%)$ of 32 acute pancreatitis patients, the process was confined within the peripancreatic region. In the remaining $31(96.8 \%)$ patients, it extended into the pararenal space. The anterior pararenal space was involved in all 31cases. In $29(90.6 \%)$ patients, there was preservation of the fat in the posterior pararenal space.

In $23(71.8 \%)$ of 32 acute pancreatitis patients, the process involved the left side only. In $7(21.8 \%)$ patients, the extensions were bilateral. In $1(3.1 \%)$ patient the renal fascial thickening was present on right side only .In remaining $1(3.1 \%)$ patient renal fascia was not thickened. The left renal fascia is more commonly affected by pancreatic inflammation than the right due to the proximity of the body and tail of the pancrease.

Ishikawa et al. (2006) described the retroperitoneal extention of acute pancreatitis. They succeeded in classifying all patterns of fluid collection in the retroperitoneum into five grades. Grade I $(n=8)$, fluid collection is confined to the anterior pararenal space or spreads into the retromesenteric plane adjacent to the anterior pararenal space; Grade II $(n=16)$, the fluid spreads further into the lateroconal plane or retrorenal plane via the retromesenteric plane; Grade III $(n=8)$, the fluid spreads further caudally into the combined interfascial plane; Grade IV ( $n=15)$, the fluid escapes from the interfascial plane into the subfascial plane via the narrow connecting passageway; Grade V $(n=11)$, the fluid intrudes directly into the posterior pararenal space across the lateroconal plane, retrorenal or subfascial plane.

Similarly, Lankisch et al. (1999) observed thickening anterior pararenal space (APS) in all 91 patients, extrapancreatic fluid collection only in anterior pararenal space (APS) in $25(28 \%)$ and in the anterior and posterior pararenal space (APPS) in $27(30 \%)$ patients. In the remaining $39(42 \%)$ patients, both the APPS and the splenic area were involved. In another study, done by Raptopouios et al. (1988), found involvement of the anterior pararenal space in all 40 . In 37 patients $(92.5 \%)$, there was preservation of the fat in the properitoneal compartment and the posterior pararenal space. In nine patients $(22.5 \%)$, the extensions were bilateral. Only three patients $(7.5 \%)$ had pancreatic phlegmon or effusion extending into the posterior pararenal space.

Dodds et al. (1986) study observed that in 10 patient's peripancreatic fluid in the left anterior pararenal space. In eight patients, a fluid collection in the anterior pararenal space extended caudally into the lower part of the iliac fossa.

On the other hand, Chintapaet at. (1982) found renal fascia thickening in $44(62 \%)$ and was not evident in $27(38 \%)$, which may be due to inclusion of chronic pancreatitic patient in their study. In 23 of the 44 cases, thickening was noted on the left side only. In 19 cases thickening of renal fascia was seen on both sides. In the remaining two, renal fascial thickening was present on right side only.

In this current study it was observed that the mean renal fascia thickness was $7.20 \pm 3.32 \mathrm{~mm}$ ranging from 1 to 13 $\mathrm{mm}$ and maximum 15(47\%) patients had thickened fascia ranging from $4.0-6.9 \mathrm{~mm}$.

In this present study, sensitivity of thickened renal fascia in the diagnosis of acute pancreatitis was found $97 \%$, specificity $50 \%$, accuracy $80 \%$, positive predictive value $77 \%$, negative predictive value $90 \%$. A study by Imamura et al. (2010) found the sensitivity 98\%, specificity 55\%, accuracy $75 \%$, positive predictive value $65 \%$ and negative predictive value $95 \%$ which was closely resembled with the current study.

In this present study, specificity of thickened renal fascia in diagnosis of acute pancreatitis is 50\% which is slightly higher than serum amylase (specificity 40\%). Thickened renal fascia can be considered as an important imaging finding when enzyme levels are normal even in severe acute pancreatitis.

\section{References}

1. Lenhart, DK \& Balthazar, EJ, 2008, 'MDCT of Acute Mild (Nonnecrotizing) Pancreatitis: Abdominal Complications and Fate of Fluid Collections, AJR, 190:643-649.

2. Hayes, PC, Simpson, KJ \& Garden, ID, Paterson-Brown ,S,(eds), Davidson's principles and practical medicine, 21thedn. Churchill Livingstone, Edinburgh, p.888.

3. Matull, WR, Pereira , SP \& O’Donohu, JW, 2006, 'Biochemical markers of acute pancreatitis, J ClinPathol, 59:340-344.4.

4. Murfitt,J,2002, The Pancreas, Sutton, D ,Robinson, PJA, Jenkins, JPR, Whitehouse ,RW \& Stevens, $\mathrm{JM}$ (eds), Textbook of radiology and imaging , 7thedn, 
Churchill Livingstone, London, p.787.

5. Imamura, Y, Hirota, M, Ida, S, Hayashi, N, Watanabe, M, Takamori, H, Awai, K\&Baba, H, 2010, 'Significance of Renal Rim Grade on Computed Tomography in Severity Evaluation of Acute Pancreatitis', Pancreas,39(1):41-6.

6. Chishty, IA, Bari, V, Pasha, S, Haider, DBH \& Rafique, Z, 2005, 'Role of Computed Tomography in Acute Pancreatitis and its Complications among Age Groups', JPMA, 55:431.

7. Tenner, S \& Steinberg, WM, 2010, Acute Pancreatitis, Feldman, M, Friedman, LS, Brandt, LJ, (eds),Gastrointestinal and liver disease.9thedn, Saunders Elsevier,Philadelphia,p. 959.
8. Ralls, P, 2006 The Pancreas, Rumack, CM, Wilson, SR, Charboneau, JW, Levine, D, (eds), Diagnostic Ultrasound, 4thedn, Elsevier, Mosboy, p.228.

9. Koo, BC, Chinogureyi, A \& Shaw, AS, 2010, 'Imaging acute pancreatitis', The British Journal of Radiology, 83:104-112.

10. Chintapa, K, Lawson, TL, Foley, WD \&Berland, LL, 1982, 'Renal fascial thickening in Pancreatitis', Journal of Computer Assisted Tomography, 6(5):983-986. 